ఠ

CASE REPORT

\title{
A fatal case of congenital pulmonary airway malformation with aspergillosis in an adult
}

This article was published in the following Dove Press journal:

International Medical Case Reports Journal

20 March 2014

Number of times this article has been viewed

\author{
Hilary A Enuh' \\ Edward L Arsura ${ }^{2}$ \\ Zaza Cohen ${ }^{3}$ \\ Keith T Diaz ${ }^{2}$ \\ Jay $M$ Nfonoyim² \\ Phillip J Cosentino ${ }^{2}$ \\ Jessie K Saverimuttu ${ }^{4}$ \\ 'Department of Internal Medicine, \\ Richmond University Medical Center, \\ Staten Island, NY, USA; ${ }^{2}$ Pulmonary \\ and Critical Care Medicine, Richmond \\ University Medical Center, Staten \\ Island, NY, USA; ${ }^{3}$ Pulmonary and \\ Critical Care Medicine, Rutgers, The \\ State University of New Jersey, New \\ Jersey Medical School, Newark, NJ, \\ USA; ${ }^{4}$ Infectious Disease Control Unit, \\ Richmond University Medical Center, \\ Staten Island, NY, USA
}

Correspondence: Zaza Cohen Division of Pulmonary and Critical Care Medicine, Rutgers, The State University of New Jersey, New Jersey Medical School, 150 Bergen Street, UH I-354, Newark, NJ 07I03, USA

Email zazacohen@yahoo.com

\begin{abstract}
Congenital cystic adenomatoid malformation, currently referred as congenital pulmonary airway malformation (CPAM), is one of the rare lung malformations seen in adults. We report a case of a 59-year-old male with a chronic cough and hemoptysis that was not amenable to bronchial embolization. Further work up revealed cystic changes with fungal ball and type 1 CPAM. Patients with this condition who survive to adulthood usually suffer from recurrent respiratory bacterial infections. Only three cases of fungal involvement have ever been described. We present a fatal case, as well as the oldest patient.
\end{abstract}

Keywords: CPAM, aspergillosis, embolization

\section{Introduction}

Congenital pulmonary airway malformation (CPAM), previously known as congenital cystic adenomatoid malformation, is an uncommon fetal lung anomaly involving cystic changes to the terminal bronchioles. Most cases of CPAM are found in neonates and infants with acute respiratory distress. Occasionally, CPAM remains unrecognized until adolescence or later life. Recurrent pulmonary infections, lung abscess, and pneumothorax are frequent features of CPAM in adults. Fungal infection of CPAM is a rare complication, and of the three previously reported cases in the literature, all have been associated with Aspergillus species. We present a fatal case of CPAM with aspergilloma, complicated by massive hemoptysis not amenable to embolization, and a review of literature.

\section{Case summary}

A 59-year-old male presented to our hospital with progressive dyspnea, productive cough, and hemoptysis of 2 weeks duration. Three days prior to admission his history was significant for massive hemoptysis, averaging $200-500 \mathrm{~mL}$ of gross blood daily. However, there was no evidence of chest pain, fever, night sweats, weight loss, recent travel, or contact with the sick, nor was there any other bleeding noted. His past medical history was significant for asthma and 30 years of smoking. He was also treated for active pulmonary tuberculosis 7 years ago. He had one similar episode of hemoptysis 1 year prior to this admission, but declined full work-up and failed to follow up after discharge.

Upon presentation, his vital signs were within normal limits, with a respiratory rate of 20 cycles/minute and oxygen saturation of $96 \%$ on room air. The lungs were clear to both percussion and auscultation, except for a decreased breath sound at the 
left upper lobe apex. Other findings on physical examination were within normal limits. Laboratory results were as follows: hemoglobin, $11.2 \mathrm{~g} / \mathrm{dL}$; hematocrit, $33.1 \mathrm{~g} / \mathrm{dL}$; white blood cell count, $5.7 \times 10^{9} / \mathrm{L}$; and a normal differential count. Basic metabolic profile, liver chemistry, and coagulation parameters were all within normal limits. A chest radiograph revealed hyperinflation, bilateral nodularity, pleural thickening with fibrocystic changes in the left apex and a possible cavitary lesion. Further work-up with a chest computed tomogram (Figure 1) revealed irregular speculated density, representing a cavitary lesion filled with mycetoma (fungal ball), numerous nodules scattered throughout both lungs and extensive bullous disease, especially at the left upper lobe. Sputum staining for acid-fast organisms was negative, but Haemophilus parainfluenzae was grown on culture media. Left upper lung biopsy revealed CPAM type 1 with aspergilloma (Figures 2 and 3). The Periodic acid-Schiff and Grocott's methenamine silver stains showed tightly packed septate hyphae. He was started on itraconazole and ceftriaxone. Due to persistent massive hemoptysis, he was referred for bronchial artery angiography and embolization. The bronchial arteriogram showed neovascularity supplying the inflammatory changes within both apices of the lungs, which were successfully embolized. Subsequently, he had some improvement of the cough and hemoptysis; however, 2 days after the embolization, massive hemoptysis recurred, which necessitated emergency surgical intervention. He had a left upper lobectomy. Intraoperatively, he developed diffuse oozing from the dissected surfaces, leading to hemodynamic instability and disseminated intravascular coagulopathy. Every effort to resuscitate him including massive transfusion of blood products was futile. He died from hemorrhagic shock secondary to left lobectomy.

\section{Discussion}

CPAM is a nonhereditary, hamartomatous cystic malformation of the lower respiratory tract first described in $1949 .{ }^{1}$ Its
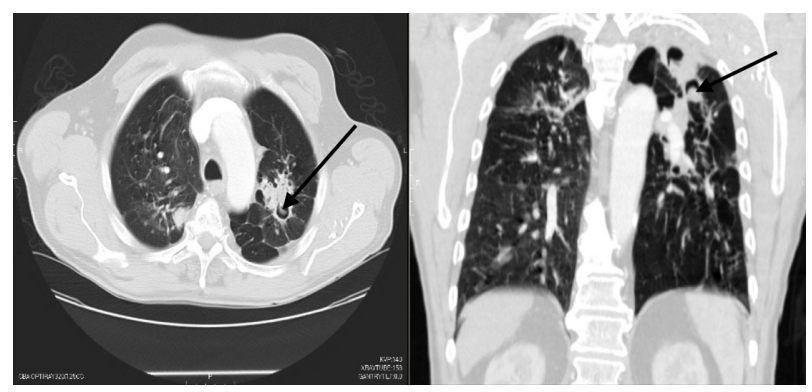

Figure I Cystic changes with mycetoma in the apex of the left lung.

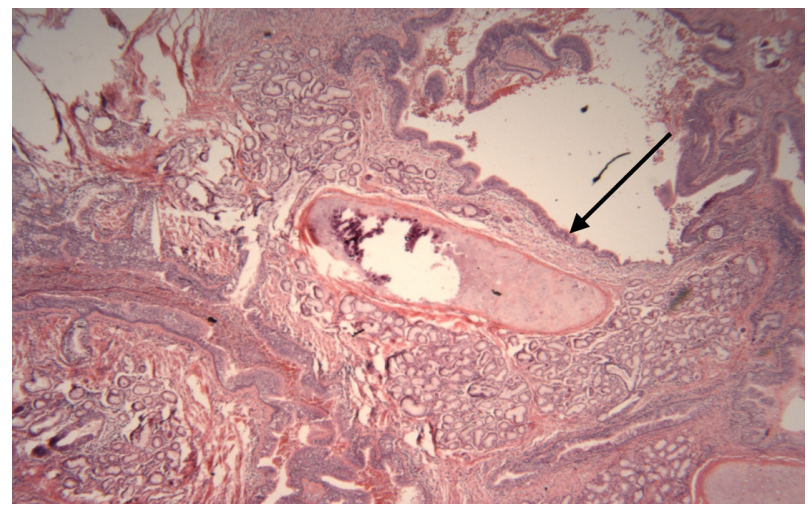

Figure 2 Region with bronchogenic cyst-like structures showing components of bronchial wall, including mucinous glands and cartilage.

incidence is estimated at 1 in 25,000-30,000 pregnancies, with most cases of CPAM found in neonates and infants with acute respiratory distress. ${ }^{2}$ In the past, only about $17 \%$ of the patients with CPAM survived to 6 months of age, ${ }^{3}$ recently more than $60 \%$ survival has been noted depending on the CPAM volume ratio. ${ }^{4}$ Patients who make it to adulthood usually have recurrent respiratory bacterial infections. CPAM was initially classified into three types, but currently, five different types have been described based on the degree of differentiation. ${ }^{5}$

1. Type 0 CPAM - This rare type with absence of alveoli is seen in neonates, and causes death in the first few hours of life.

2. Type I CPAM - This is the most common type, which was also seen in our patient. It is a localized cystic lesion which typically affects only a part of a lobe and can be surgically resected. It has the best prognosis of all the types of CPAM. The cysts are lined by pseudostratified ciliated columnar epithelium, interspersed with rows of mucus cells.

3. Type II CPAM - The lesion is sponge-like and consists of multiple small cysts as well as solid areas. It is lined by

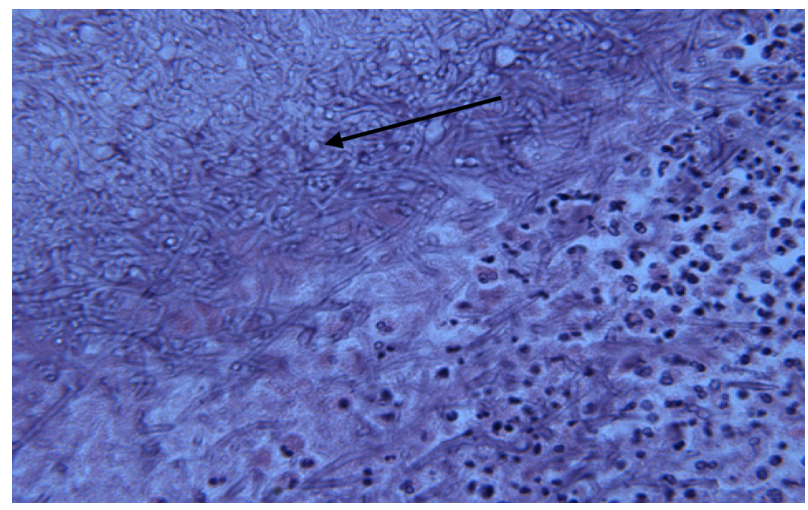

Figure 3 Clusters of Aspergillus hyphae. 
bronchiolar epithelium and separated by poorly developed alveoli.

4. Type III CPAM - This is an uncommon subtype which presents in infancy, almost exclusively in male babies. These are large bulky lesions that typically involve and expand the entire lobe of lung.

5. Type IV CPAM - These are peripheral cysts with thin walls, which may undergo malignant transformation to pleuropulmonary blastoma.

Our case met the diagnostic criteria of type I for its distinct pathologic change affecting only the left upper lobe containing multiple cysts, the largest measuring $5 \mathrm{~cm}$ in diameter, lined by pseudostratified epithelium interspersed with mucus cells. Aspergillus species are fungal molds spread via aerosolized spores. They are ubiquitous, causing a variety of illnesses in immunocompetent patients ranging from asthma, extrinsic allergic alveolitis, to allergic bronchopulmonary aspergillosis. It can cause serious systemic illness in immunocompromised patients like invasive pulmonary infection and extra pulmonary dissemination. Aspergilloma originates most often in residual cavitary lesions from prior pulmonary tuberculosis, which our patient had, although it can occur in patients with any cavitary lung disease. ${ }^{6}$ However, aspergilloma has rarely been described in association with CPAM. Our case study is the fourth case ever described as well as the oldest. The first case was described in an 18-year-old girl with type II CPAM, presenting with recurrent hemoptysis. Another case was described in a 14-year-old male with recurrent cough and mild hemoptysis. ${ }^{8}$ Feng et al reported a case of aspergilloma in CPAM type II in a 26-year-old female with persistent cough. ${ }^{9}$ An aspergilloma can exist for years without symptoms. Hemoptysis is the most common manifestation and can be fatal, as seen in our patient.

Although typical CPAM structure could be ruined through chronic aspergillosis or tuberculosis, in this case we do have areas showing juxtaposed glands lined with respiratory epithelium separated by orderly interstitial tissue (Figures 2 and 4). The orderly structure of the interstitial tissue indicates that the glands are not brought together by post inflammatory scarring. The intact mucinous glands also argue against destructive inflammation around the glands. Furthermore, no Rasmussen's aneurysm, which can also mimic this presentation, was seen on autopsy of this patient. Surgical resection is the mainstay of treatment for symptomatic patients with simple aspergilloma, whereas medical therapy has limited activity in treatment of aspergilloma. Bronchial artery embolization, a temporizing procedure in a patient with life-threatening hemoptysis, ${ }^{10}$

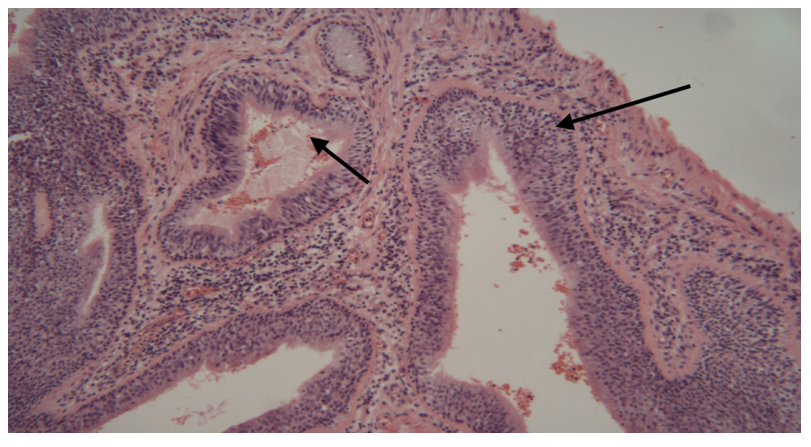

Figure 4 Juxtaposed glandular structures with orderly interstitial tissues with moderate chronic inflammation. The lining epithelium is respiratory pseudostratified columnar type. No accompanying arteries are seen.

was used in our patient with a nonsatisfactory response. In the cases described by Feng et $\mathrm{al}^{9}$ and Yonker et $\mathrm{al},{ }^{8}$ the patients were successfully treated with surgery, owing to their age and milder stage of the disease. Surgery is generally well tolerated in patients with a single aspergilloma, with a mortality rate of $<1$ percent. ${ }^{11}$ However, patients with more complicated diseases, such as chronic cavitary pulmonary aspergillosis (eg, as seen in our patient) or pleural-based aspergillomas, are more likely to have complications, and postoperative deaths of $5.7 \%$ have been recorded in such groups. ${ }^{12}$ This group of people may benefit from antifungal therapy; itraconazole and voriconazole are the preferred oral agents for the treatment of chronic pulmonary aspergillosis.

Aspergilloma is a rare complication of CPAM and requires early surgical intervention. It can be fatal if complicated with chronic cavitary pulmonary aspergillosis.

\section{Acknowledgments}

We are grateful to Dr Minsheng Zhuang, the Clinical pathologist who reviewed the figures included in this paper. A written consent was obtained from the patient's next of kin to report this case.

\section{Disclosure}

The authors report no conflicts of interest in this work.

\section{References}

1. Ch'In KY, Tang MY. Congenital adenomatoid malformation of one lobe of a lung with general anasarca. Arch Pathol (Chic). 1949;48:221-229.

2. Laberge JM, Flageole H, Pugash D, et al. Outcome of the prenatally diagnosed congenital cystic adenomatoid lung malformation: a Canadian experience. Fetal Diagn Ther. 2001;16:178-186.

3. LacknerRP, Thompson III AB, Rikkers LF, Galbraith TA. Cystic adenomatoid malformation involving an entire lung in a 22-year-old man. Ann Thorac Surg. 1996;61:1827-1829.

4. Fetal Care Center of Cincinnati. Available from: http://www.cincinnatichildrens.org/service/f/fetalcare/conditions/ccam/detail/. Accessed December 22, 2013. 
5. Stocker JT. Congenital pulmonary airway malformation: a new name and an expanded classification of congenital cystic adenomatoid malformation of the lung. Histopathology. 2002;41:424-431.

6. Joynson DH. Pulmonary aspergilloma. Br J Clin Pract. 1977;31: 207-216.

7. Pedro-Botet ML, Olazabal A, Astudillo J, Sopena N, Modol JM, Sabria M. Cavitating lung lesion and hemoptysis in a young woman. European Society of Clinical Microbiology and Infectious Diseases. 2000;6(5):233-288.

8. Yonker LM, Mark EJ, Canaperi CA. Aspergilloma in a patient with an occult congenital pulmonary airway malformation. Pediatr Pulmonol. 2012;47(3):308-310.
9. Feng A, Cai H, Sun Q, Zhang Y, Chen L, Meng F. Congenital cystic adenomatoid malformation of lung in adults: 2 rare cases report and review of the literature. Diagnostic Patholog. 2012;7:37.

10. Kauffman CA. Quandary about treatment of aspergillomas persists. Lancet. 1996;347:1640.

11. Brik A, Salem AM, Kamal AR, et al. Surgical outcome of pulmonary aspergilloma. Eur J Cardiothorac Surg. 2008;34(4):882.

12. Regnard JF, Icard P, Nicolosi M, et al. Treatment of chronic pulmonary aspergillosis. Ann Thorac Surg. 2000;69(3):898.

\section{Publish your work in this journal}

The International Medical Case Reports Journal is an international, peer-reviewed open-access journal publishing original case reports from all medical specialties. Previously unpublished medical posters are also accepted relating to any area of clinical or preclinical science. Submissions should not normally exceed 2,000 words or
4 published pages including figures, diagrams and references. The manuscript management system is completely online and includes a very quick and fair peer-review system, which is all easy to use. Visit http://www.dovepress.com/testimonials.php to read real quotes from published authors.

Submit your manuscript here: http://www.dovepress.com/international-medical-case-reports-journal-journal 